\title{
Effects of Weight Loss on Vascular Function in Obese Individuals with Poor Cardiovascular Health: Design and Research Protocol of an Interventional Clinical Trial
}

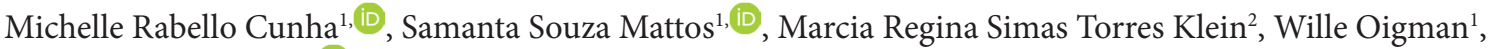 \\ Mario Fritsch Neves ${ }^{1, *,(i D)}$ \\ ${ }^{1}$ Department of Clinical Medicine, State University of Rio de Janeiro, Ave.Vinte e Oito de Setembro, 77 room 329, Vila Isabel, Rio de Janeiro, RJ 20551-030, Brazil \\ ${ }^{2}$ Department of Applied Nutrition, Nutrition Institute, State University of Rio de Janeiro, Rua São Francisco Xavier, 524, Pavilhão João Lyra Filho, \\ 12th floor, room 12026D, Maracanã, Rio de Janeiro, RJ 20550-900, Brazil
}

\section{ARTICLE INFO}

Article History

Received 22 December 2019 Accepted 23 July 2020

Keywords

Obesity

weight loss

cardiovascular disease

arterial stiffness

endothelial function

obstructive sleep apnea

\begin{abstract}
Introduction: Excessive adipose tissue is associated with adverse metabolic effects and is an important risk factor for chronic diseases. According to the American Heart Association, most cardiovascular events can be prevented by adhering to healthy practices, reflecting cardiovascular health as poor, intermediate, and good.

Objective: To evaluate the effect of weight loss on vascular function, metabolic profile and inflammatory markers of obese subjects with poor cardiovascular health.

Methods: Obese individuals, both sexes, aged 40-70 years, will be instructed to follow a hypocaloric diet ( $-800 \mathrm{Kcal} / \mathrm{day}$ ) for 16 weeks. Before and after intervention, participants will undergo clinical, nutritional (anthropometry and dual energy X-ray absorptiometry), biochemical, vascular and sleep evaluation. Patients will be submitted to sympathetic tone assessment using a frequency meter Polar ${ }^{\oplus S 800}$ (Polar Electro OY, Kempele, Finland), oscillometric pulse wave analysis by Mobil-O-Graph ${ }^{\oplus}$ (IEM, Stolberg, Germany), post-occlusive microvascular reactivity by laser speckle contrast imaging, carotid ultrasound and degree of obstructive sleep apnea through the home polysomnography device (WatchPAT $200^{\circ}$ ). Serum levels of leptin, adiponectin, interleukin-6, tumor necrosis factor-alpha and C-reactive protein will be determined. Participants will be stratified in two groups according to cardiovascular health (poor vs. intermediate/ideal). Cardiovascular health is said to be ideal by the presence of optimal health behaviors (non-smokers, adequate body mass index, physical activity level and healthy eating pattern) and health factors (blood pressure, total cholesterol and blood glucose). Ideal cardiovascular health is considered for those with five or more metrics within this qualification, intermediate for presence of three or four metrics and poor for two or less metrics.
\end{abstract}

(C) 2020 Association for Research into Arterial Structure and Physiology. Publishing services by Atlantis Press International B.V. This is an open access article distributed under the CC BY-NC 4.0 license (http://creativecommons.org/licenses/by-nc/4.0/).

\section{INTRODUCTION}

The World Health Organization (WHO) points out obesity as a major public health problem worldwide $[1,2]$. According to the WHO report, $39 \%$ of adults aged 18 years and over were overweight in 2016, and 13\% were obese [3]. This increase in obesity prevalence is alarming since it is associated with adverse metabolic effects and could enhance the risk for chronic diseases such as type 2 diabetes mellitus, hypertension and dyslipidemia [4,5]. The risk of ischemic heart disease and cerebrovascular events increases with the rise in Body Mass Index (BMI). In addition, BMI can predict global mortality, given that survival reduces as BMI increases [6]. Thus, obesity has a negative impact on morbidity and mortality, increasing the cost of healthcare $[2,7]$.

"Corresponding author. Email: mariofneves@gmail.com

Peer review under responsibility of the Association for Research into Arterial Structure and Physiology

Data availability statement: The data that support the findings of this study are available from the corresponding author, MFN, upon reasonable request.
Body fat is currently seen as an endocrine organ, producing several bioactive substances known as adipokines and containing various cell types including adipocytes, pre-adipocytes and macrophages. Inflammatory responses at the cellular and molecular levels are more exacerbated in obese patients, favoring the occurrence of structural and functional vascular alterations, leading to an increase in cardiometabolic risk [8,9]. This inflammatory state is mediated by imbalance in cytokine and adipokine production, with increased expression of proinflammatory factors, such as leptin, Interleukin-6 (IL-6) and Tumor Necrosis Factor-alpha (TNF- $\alpha$ ), and reduced levels of anti-inflammatory factors, including adiponectin and IL-10, promoting metabolic disorders and supporting vascular changes and blood pressure elevation [10].

It is well known that Obstructive Sleep Apnea (OSA) is related to elevated BMI and is considered an independent risk factor for Cardiovascular Disease (CVD) [11]. Several factors contribute to the development of OSA in obese subjects including narrowing of the upper airways by fat deposition in the peripheral region, and the release of humoral mediators and inflammatory cytokines by 
adipose tissue [12]. A previous study suggests that weight loss may reduce the severity of OSA, with a consequent reduction in CVD risk [13].

A lifestyle intervention in obese people has been considered a useful approach to decrease cardiovascular risk by improving nutritional habits and stimulating physical activities [14]. Treatment for obesity includes adherence to a healthy lifestyle, with nutritional monitoring of low-calorie diets. The minimum percentage of weight loss considered clinically useful varies between $5 \%$ and $10 \%$ in order to improve associated conditions [4].

According to the American Heart Association (AHA), most cardiovascular events could be avoided by engaging in healthy activities. The AHA defined that some behaviors, such as smoking, BMI, physical activity, healthy dietary score, total cholesterol, blood pressure and fasting glucose could be considered as health metrics reflecting poor, intermediate and ideal cardiovascular health rating $[15,16]$.

Epidemiological studies have indicated that a greater number of health behaviors was identified as a risk reduction factor for mortality and incidence of CVD. Maintaining adequate body weight and other health metrics in communities could reduce populationlevel mortality $[17,18]$.

In the current context of high prevalence of obesity and its impact on morbidity and mortality, weight loss is an effective alternative for metabolic, inflammatory, vascular and quality of life improvement. To date, no clinical trial was conceived to determine the effects of weight loss on metabolic, inflammatory and vascular changes in obese individuals with poor cardiovascular health. Thus, this study hypothesis is that weight loss in obese people with poor cardiovascular health will result in more vascular, metabolic and inflammatory benefits than in those with intermediate or ideal cardiovascular health. It will be also considered whether the presence of OSA influences these benefits.

The general objective of this study is to assess the effects of weight loss on vascular function in obese individuals with poor cardiovascular health. As specific endpoints, we will evaluate the total and central body adiposity, peripheral and central pressure, arterial stiffness, endothelial function, Apnea-Hypopnea Index (AHI), insulin resistance and inflammatory markers, before and after weight loss.

\section{MATERIALS AND METHODS}

\subsection{Study Design}

This will be a prospective intervention study to be performed in an outpatient clinic at State University of Rio de Janeiro, Brazil. Patients with grade 1 and 2 obesity will undergo 16-week hypocaloric nutritional intervention to promote weight loss.

Included patients will make five visits during the study. In the first visit (V1), obese individuals will be invited to participate voluntarily in the project as they meet the inclusion criteria and after reading and signing of the Informed Consent Form (ICF). Afterward, the participants will be allocated into two groups according to the cardiovascular health classification (poor vs. intermediate/ideal) and will be submitted to clinical, biochemical and nutritional evaluation. Patients will return after 1 week (V2) for assessment of vascular structure and function. On the same day, the participants will receive the device that will evaluate the presence and degree of OSA. On the following day (V3), study participants will return the device and will receive instructions to follow a hypocaloric nutritional intervention. After 8 weeks of intervention, patients will return for a new clinical and nutritional assessment (V4). Every 4 weeks, phone contacts will be made to keep the patient motivated and to clarify possible doubts. At the last visit (V5), patients will undergo a new clinical, laboratory, nutritional, OSA, structural and functional vascular evaluation (Figure 1). The protocol was approved by the local Ethics Committee and registered at ClinicalTrials.gov (NCT04198519). All the participants will read and sign the ICF.

\subsection{Inclusion Criteria}

The study population will consist of outpatient men and women, aging $40-70$ years, with BMI $\geq 30$ and $<40 \mathrm{~kg} / \mathrm{m}^{2}$, habitual food consumption maintained for at least 4 weeks, and after signature of the ICF.

\subsection{Exclusion Criteria}

Patients will be excluded if presenting Systolic Blood Pressure (SBP) $\geq 160 \mathrm{mmHg}$ and/or Diastolic Blood Pressure (DBP) $\geq 100 \mathrm{mmHg}$,

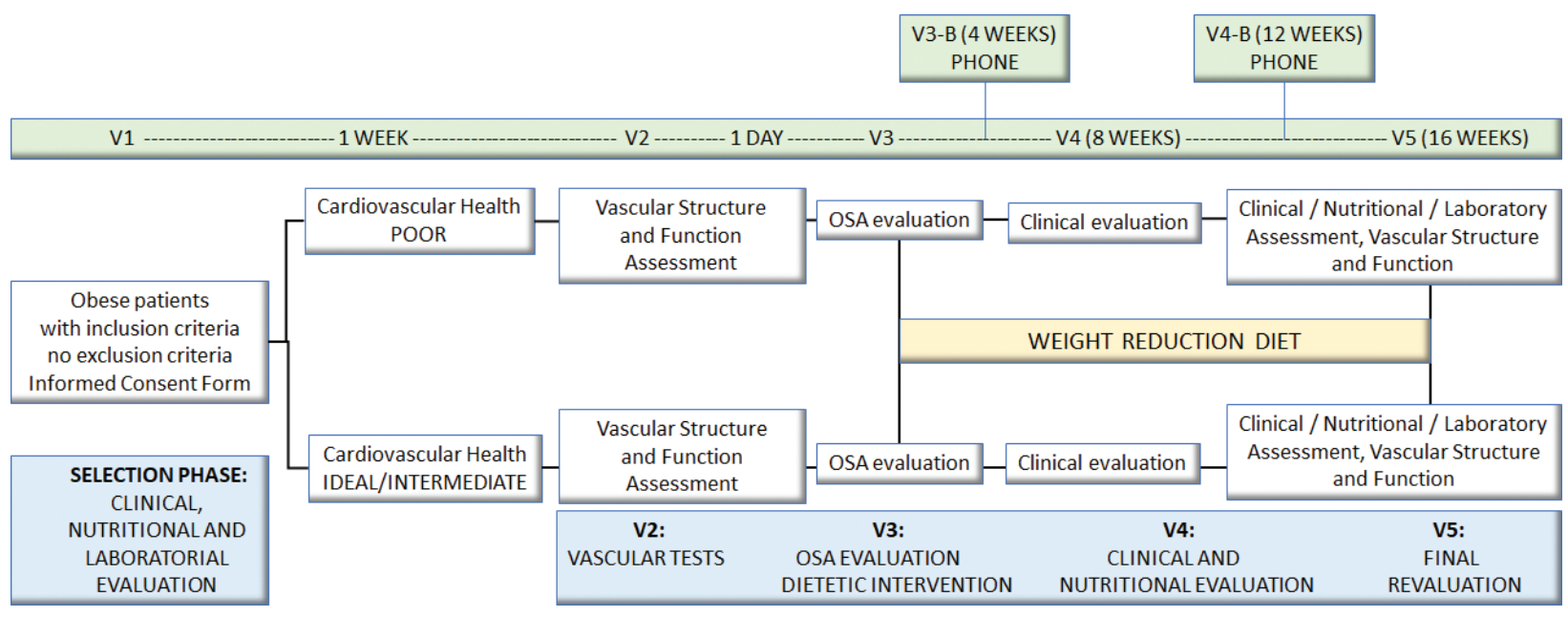

Figure 1 Study design. 
diabetes mellitus, hormone replacement therapy, evidence of secondary hypertension or clinically evident changes in thyroid function. In addition, acute or chronic kidney or liver disease, history of cancer in the last 5 years and coronary artery disease clinically evident will also be considered exclusion criteria.

\subsection{Sample Size Assessment}

There are no studies with similar objectives, methods and outcomes to enable more accurate sample size calculation. In fact, there are no studies in the literature evaluating the effects of weight loss on endothelial function measured by post-occlusive reactive hyperemia using laser speckle contrast image. However, to determine sample size for this study, we considered the variation in flow-mediated dilatation as the primary intervention outcome based on weight reduction and the results of the studies by Angelico et al. [19]. Thus, considering a study power of $80 \%$, a significance level of $5 \%$, and a $10 \%$ dropout rate, at least 53 patients will be required in the study.

\subsection{Cardiovascular Health}

In 2010, AHA established a set of seven health behaviors and factors that were considered ideal for good cardiovascular health. This concept has the impact goal of reducing CVD and stroke deaths by $20 \%$ by 2020 . Cardiovascular health is said to be good by the presence of optimal health behaviors (non-smokers, adequate BMI, physical activity level and healthy eating pattern) and ideal health factors (blood pressure, total cholesterol and blood glucose), as shown in Tables 1 and 2. Cardiovascular health will be defined by a score ranging from 0 to 14 . Poor cardiovascular health will range from 0 to 6 , intermediate from 7 to 11 and ideal for scores between 12 and 14 [16].

\subsection{Clinical Evaluation}

Clinical history data will be collected to assess date of birth, smoking and alcoholism status, concomitant diseases and medications in use. The level of physical activity will be determined using the International physical activity questionnaire, validated in Brazil by Matsudo et al. [20] and will be applied at the beginning (V1) and at the end of the study (V5). The anthropometric measurements will be performed by two experienced dietitians. Height will be measured using a stadiometer accurate to $\pm 0.5 \mathrm{~cm}$ and weight will be obtained with a digital scale, accurate to $\pm 0.1 \mathrm{~kg}$ (Filizola S.A., São Paulo, SP, Brazil) according to the techniques recommended by WHO. BMI will be calculated using the standard equation $\left(\mathrm{kg} / \mathrm{m}^{2}\right)$ [21]. A flexible and inextensible fiberglass tape measure will be used to obtain waist, hip and neck circumferences. Waist Circumference (WC) will be measured in the standing position midway between the lowest rib and the iliac crest, at mid-exhalation. Hip Circumference (HC) will be measured at the widest point over de hip/buttocks with the tape parallel to the floor. Neck circumference will be measured in the midway of the neck between mid-cervical spine and mid-anterior neck to $0.5 \mathrm{~cm}$, if palpable, just below the laryngeal prominence [22]. Waist-to-Hip Ratio (WHR) will be obtained by dividing the WC $(\mathrm{cm})$ by $\mathrm{HC}$ $(\mathrm{cm})$ and waist-to-Height Ratio (WHtR) by dividing WC $(\mathrm{cm})$ by height $(\mathrm{cm})$.which will allow the calculation of WH and WHtRs.

\subsection{Body Composition Assessment}

Body composition will be assessed by dual-energy radiological absorptiometry, which will be performed by a trained technician using GE Medical Systems Lunar ${ }^{\varpi}$ (Madison, WI, USA), with the participant in the supine position. The total amount of fat mass and lean tissue and bone mineral mass will be estimated with enCore 2008 version 12.20 software (GE Medical Systems, Madison, WI, USA). Body composition will be evaluated in whole body and different sites, such as trunk. Visceral adipose tissue analysis will be performed by CoreScan VAT (validated using computed tomography), a software that evaluates visceral fat (mass in $g$ and volume in $\mathrm{cm}^{3}$ ) in the android region.

\subsection{Biochemical Tests}

Venous blood samples will be collected in a refrigerated room, with patients fasting for $12 \mathrm{~h}$. Hemogram, glycated hemoglobin, plasma glucose, insulin, creatinine, Total Cholesterol (TC), Triglyceride

Table 2 Healthy dietary pattern considering $2000 \mathrm{kcal} /$ day [18]

\begin{tabular}{ll}
\hline Food & Consumption pattern \\
\hline Fruits and vegetables & $\geq 4.5 \mathrm{cups} /$ day \\
Fish & $>2$ servings/week \\
Whole grains & $>1.1 \mathrm{~g} \mathrm{fiber} / 10 \mathrm{~g}$ carbohydrate \\
Sodium & $<1500 \mathrm{mg} /$ day \\
Sweetened beverages & $<450 \mathrm{kcal} /$ week (or one-fourth of nutritional needs) \\
\hline
\end{tabular}

Table 1 Poor, intermediate, and ideal cardiovascular health definition for each metric [18]

\begin{tabular}{|c|c|c|c|}
\hline Metric & Poor (0 point) & Intermediate (1 point) & Ideal ( 2 points) \\
\hline Current smoking & Yes & Stopped $\leq 12$ months & Never or stopped $>12$ months \\
\hline Body mass index $\left(\mathrm{kg} / \mathrm{m}^{2}\right)$ & $\geq 30$ & $25-29.9$ & $<25$ \\
\hline Physical activity & None & $\begin{array}{l}\text { 1-149 } \mathrm{min} / \text { week moderate intensity } \\
\text { or } 1-74 \mathrm{~min} / \text { week vigorous }\end{array}$ & $\begin{array}{l}\geq 150 \mathrm{~min} / \text { week moderate intensity or } \\
\quad \geq 75 \mathrm{~min} / \text { week vigorous }\end{array}$ \\
\hline Healthy dietary pattern ${ }^{*}$ (components) & $0-1$ & $2-3$ & $4-5$ \\
\hline Total cholesterol (mg/dl) & $\geq 240$ & $200-239$ & $<200$ \\
\hline Blood pressure (mmHg) & $\mathrm{SBP} \geq 140$ or $\mathrm{DBP} \geq 90$ & SBP $120-139$ or DBP $80-89$ & $\mathrm{SBP}<120$ or $\mathrm{DBP}<80$ \\
\hline Fasting blood glucose (mg/dl) & $\geq 126$ & $100-125$ & $<100$ \\
\hline
\end{tabular}


(TG), and High-Density Lipoprotein (HDL)-cholesterol will be evaluated. Low-Density Lipoprotein (LDL)-cholesterol will be calculated by Friedewald's formula: $\mathrm{LDL}=\mathrm{TC}-\mathrm{HDL}-\left(\frac{\mathrm{TG}}{5}\right)$ [23]. The serum levels of adipokines (leptin, adiponectin, IL-6, and TNF- $\alpha$ ) will be determined using Luminex Method. Serum C-reactive protein concentration will also be determined by turbidimetric method, considering reference value $>0.10 \mathrm{mg} / \mathrm{dl}$ as high cardiovascular risk. The insulin resistance index will be obtained using the homeostatic model assessment-insulin resistance calculation, and the cutoff point of 2.71 used to identify insulin resistance [24].

\subsection{Blood Pressure Measurement}

An electronic device (model HEM-705CP, OMRON Healthcare Inc., IL, USA), using appropriate cuff placed about $2 \mathrm{~cm}$ above the cubital fold, will be used to measure SBP and DBP in the sitting position. After six measurements at 1-min interval, standard deviation, coefficient of variation (standard deviation/mean) and amplitude (maximum - minimum) will be obtained as markers of blood pressure variability. SBP minus DBP will be calculated to determine Pulse Pressure (PP), which will allow the estimation of mean arterial pressure $=\mathrm{PP} / 3+\mathrm{DBP}$.

\subsection{Assessment of Sympathetic Tone}

Individuals will be evaluated according to the criteria for Heart Rate Variability (HRV) recommended by the European Society of Cardiology and the American Society of Pacemakers and Electrophysiology, resting at supine position, using a 20-min period with the initial $15 \mathrm{~min}$ of stabilization and the next $10 \mathrm{~min}$ of registration. All data will be processed using free software Kubios HRV (Finland). From the frequency recorder (Polar ${ }^{\circledR}$ RS800, Polar Electro OY, Kempele, Finland) the intervals between each heartbeat ( $R$ wave) will be identified, called $R-R$ intervals. In the frequency domain, Low Frequency (LF) and High Frequency (HF) components will be considered for calculations. For the analysis, the following parameters will be observed: LF, HF and the ratio between $\mathrm{LF}$ and HF, representing the sympathovagal balance (LF/HF) in absolute units $\left(\mathrm{ms}^{2}\right)$. LF and HF are often interpreted as indicators of sympathetic and parasympathetic activity, respectively [25].

\subsection{Cardiovascular Risk Estimation}

The vascular age calculation will be the same as used in the study of D'Agostino who assessed CVD risk based on the Framingham Heart Study [26], considering the estimated 10-year risk of some cardiovascular events such as myocardial infarction, cerebrovascular disease, atherosclerosis in lower limbs or cardiac failure. The population involved in this evaluation were individuals of both sexes, between 30 and 74 years old, without previous CVD. According to the number of points, in different tables for men and women, the percentage of CVD risk at 10 years is obtained and related with another table of vascular age.

The cardiometabolic age will be accessed at https://myhealthcheckup. com. This risk calculator is supported by the Canadian Institute for Health Research, developed by physicians and statisticians at McGill University in Canada. The calculation was validated in 2015 using data from the 2003-10 National Health and Nutrition Examination Survey and comparing life expectancy for Americans with data from the Framingham Heart Study [27]. The risk estimate for diabetes and CVD takes into account gender, age, ethnicity, height, weight, waist circumference, TC, LDL-cholesterol, HDL-cholesterol, SBP and DBP, use of antihypertensive medication, blood glucose, medical history of CVD, diabetes, family history of diabetes and premature CVD, performance of physical activity, and smoking [28].

\subsection{Sleep Study}

The WatchPAT 200 (WP200; Itamar Medical Ltd, Caesarea, Israel) is a four-channel Peripheral Arterial Tone (PAT)-based device with three additional channels: heart rate (PAT-based), pulse oximetry, and actigraphy. The PAT signal processes the change in arterial pulse volume, and thus measures sympathetic nerve activity. WP $200^{\circ}$ can identify apnea or hypopnea events by detecting the onset of sympathetic activity coupled with interruption of respiratory movements. Respiratory events are uncovered by a combination of PAT signal drop, Heart Rate (HR) increase, and changes in arterial oxygen saturation. At home, just before bedtime, patients place the device on the wrist of the non-dominant hand, the PAT and the oximetry channel on the second finger. Before turning off the light, the patient presses the WP $200^{\circ}$ button to begin the study. The next morning, he/she returns the device. The most important outcomes include AHI, estimated total sleep time, REM sleep percentage, oxygen desaturation index, and the lowest oxygen saturation value $[29,30]$.

\subsection{Oscillometric Pulse Wave Analysis}

Central hemodynamics will be evaluated with the Mobil-O-Graph device (I.E.M. GmbH, Stolberg, Germany). This device has been duly validated for use in scientific research by the European Society of Hypertension. This is a non-invasive method that checks the pulse wave, permitting the measurement of peripheral and central blood pressure, Pulse Wave Velocity (PWV) and markers of pulse wave reflection, such as Augmentation Pressure (AP) and Augmentation Index (AIx). Recordings are acquired by using appropriate obese adult cuffs and a high-fidelity pressure sensor MPX50550 (Freescale Inc., Tempe, AZ, USA). The following variables will be collected: central systolic pressure and pulse pressure, AP, AIx, AIx@75 (AIx corrected for heart rate of $75 \mathrm{bpm}), \mathrm{PWV}$ and arterial age.

\subsection{Microvascular Reactivity}

Endothelial function will be assessed by laser speckle contrast image (PSI Pericam System, Perimed, Sweden) evaluating Post-occlusive Reactive Hyperemia (PORH) for physiological evaluation, not invasive and continuous cutaneous endothelium-dependent microvascular perfusion changes expressed in arbitrary perfusion units [31]. The image acquisition rate will be one image/s and the distance between the laser head and the skin surface is fixed at $20 \mathrm{~cm}$. A sphygmomanometer will be used to apply a pressure of $50 \mathrm{mmHg}$ above SBP for $3 \mathrm{~min}$. Flow variations between baseline and after the 3-min occlusion period (PORH) will be registered to assess microvascular reactivity. The manufacturer's software (PIMSoft, Perimed) will be used to analyze the images. 


\subsection{Carotid Ultrasound}

The possible presence of atheromatous plaques and the measurement of the Intima-media Thickness (IMT) will be evaluated in an area without atheromatous plaques, in the common carotid artery. The right and left carotid arteries will be examined with a high-resolution ultrasound device Philips, Affiniti 70 (Philips, Andover, MA, USA) equipped with a $10-\mathrm{MHz}$ linear transducer and patients will be examined in supine position with their head in slight contralateral rotation. The same trained sonographer will perform all examinations. The region located up to $2 \mathrm{~cm}$ proximal to the beginning of the carotid bulb will be used to measure IMT. Measurements will be made at the end of diastole, identified by simultaneous electrocardiographic recording. The mean values of IMT in this length of $20 \mathrm{~mm}$ will be calculated. The cutoff point of carotid IMT $\geq 1.5 \mathrm{~mm}$ will determine the presence of atheromatous plaques.

\subsection{Assessment of Food Intake}

The assessment of food intake will be performed at V1 and V5 through one interviewer-administered $24 \mathrm{~h}$ dietary recall and a semiquantitative Food Frequency Questionnaire (FFQ). This FFQ was developed for the Brazilian population and was validated against more accurate methods to assess dietary intake [32]. Every 4 weeks during the study period, food intake will be evaluated by a 24 -h dietary recall. In weeks 4 and 12 dietary recall will be obtained through a telephone call and in week 8 (V4) the recall will be applied in person. At V1, the assessment of food intake will be used to evaluate habitual dietary intake and at weeks 4, 8 (V4), 12 and 16 (V5) to evaluate dietary adherence. Data from the $24 \mathrm{~h}$ dietary recall and from the FFQ will be analyzed using NutWin (Unifesp, SP, Brazil) and SAS software (SAS Institute Inc., Cary, North Carolina, USA) respectively, and the Brazilian Table of Food Composition.

\subsection{Dietary Intervention}

The study participants will be instructed to follow a hypoenergetic diet during all the study period and no food will be provided. The Total Energy Value (TEV) of the energy-restricted diet prescribed for each study participant in V3 will be determined by subtracting $800 \mathrm{kcal} /$ day from the total daily energy expenditure, which will be estimated using Dietary Reference Intake equations with current body weight [33]. The objective of this prescription is to reach a $5-10 \%$ weight reduction. The distribution of macronutrients will be as follows: protein $15-20 \%$ of TEV, lipids $25-30 \%$ of TEV and carbohydrates $50-60 \%$ of TEV. At V4, TEV of the diet will be adjusted according to the participant's body weight at this visit, as an attempt to maintain weight loss. At weeks 4, 8 (V4) and 12, participants will be asked about possible doubts regarding dietary prescription and will be encouraged to continue following the dietary intervention for weight loss.

\subsection{Statistical Analysis}

Results will be presented as mean \pm standard deviation. In intragroup analyzes, the paired $t$-test will be performed to compare the final and initial results in each group. The comparison between groups will be performed by Student's $t$-test for normal distribution samples and Mann-Whitney for non-Gaussian samples. The comparative analysis between groups for categorical variables will be performed by Fisher exact test. Pearson coefficient will be identified in correlation analysis between normal numerical variables and the Spearman coefficient for non-normal variables. For all analyzes, 95\% confidence interval will be adopted and the $p$-value $<0.05$ will be considered statistically significant. Results will be obtained with Statistical Package for the Social Sciences SPSS version 22.0 software (IBM SPSS Statistic, USA).

\section{DISCUSSION}

Cardiovascular disease is the most important cause of death worldwide, and it is also associated with increased morbidity. Therefore, some changes in lifestyle are commonly necessary in the approach for cardiovascular prevention. Previous studies indicate that a lower risk of CVD incidence and mortality is associated with a greater number of ideal cardiovascular health behaviors [34].

Compliance with seven health metrics that are used to obtain the cardiovascular health score can modify risk factors, and therefore can be considered primary prevention goals. Individuals presenting a greater number of ideal health metrics usually have a lower risk of cardiovascular and metabolic diseases than those with lower health scores [35].

In addition, some studies have suggested that overweight and obese individuals often have a harmful behavior profile, which may increase the risk of cardiovascular events. Therefore, lifestyle changes appear to be the main strategic approach to effectively reduce cardiovascular risk, lowering obesity-related mortality $[36,37]$.

In the present study, the authors anticipate that nutritional intervention through a low-calorie diet aimed at promoting weight loss may bring benefits such as improvement in body composition, vascular function and structure, blood pressure levels, insulin sensitivity and consequently lead to optimal cardiovascular health and reduce cardiovascular risk.

To the best of our knowledge, studies assessing cardiovascular health in the Latin American population using the AHA criteria are scarce [38]. It is believed that this concept can be very useful to encourage the achievement of ideal goals and, consequently, improve the health of the population, especially in stimulating weight loss $[15,35]$.

Our study hypothesis is that weight loss in obese people with poor cardiovascular health will result in greater vascular, metabolic, inflammatory benefits when compared to those with intermediate or ideal cardiovascular health.

Some limitations must be considered in this study. Good compliance to nutritional prescription is crucial to reach a $5-10 \%$ weight reduction, and patients with poor cardiovascular health usually do not follow dietary recommendations. However, the research team will have access to all the participants to stimulate the compliance to the protocol. This approach can also avoid the loss of follow-up, considering the 16-week duration of this study. There may also be difficulty balancing the distribution of patients into study groups, as obese people tend to present a poor cardiovascular health. 
This is the main reason we included intermediate and ideal cardiovascular health in the same group. As PWV will be provided by Mobil-O-Graph, a device that uses an algorithm based on age and $\mathrm{BP}$, this parameter will not be an independent variable, being a possible limitation for study conclusions.

The prospective interventional design and its pioneering focus can be considered strengths of this study. The planned research is an evaluation of dietary interventions on cardiovascular health. To date, no trials have examined the effects of weight loss on vascular functions in obese individuals with different scores of cardiovascular health. Further studies are warranted to improve our understanding of the magnitude of this problem in obese adults and to elucidate treatable risk factors.

\section{CONFLICTS OF INTEREST}

On behalf of all authors, the corresponding author declares no conflicts of interest.

\section{AUTHORS' CONTRIBUTION}

MRC, MRSTK, WO and MFN formulated this randomized clinical trial. MRSTK and MFN coordinate the study. MRC, MRSTK and MFN supervise the study. MRC and SSM will collect the data. MRC, SSM, MRSTK and MFN will do the statistical analysis.

\section{FUNDING}

MFN received a grant from Carlos Chagas Filho Foundation for Research Support at the State of Rio de Janeiro (FAPERJ) and from the National Council for Scientific and Technological Development $(\mathrm{CNPq})$ for funding this project.

\section{ACKNOWLEDGMENTS}

We thank Mrs Claudia Deolinda Lopes Alves Madureira for all the support for this study.

\section{REFERENCES}

[1] Ng M, Fleming T, Robinson M, Thomson B, Graetz N, Margono $\mathrm{C}$, et al. Global, regional, and national prevalence of overweight and obesity in children and adults during 1980-2013: a systematic analysis for the Global Burden of Disease Study 2013. Lancet 2014;384:766-81.

[2] Upadhyay J, Farr O, Perakakis N, Ghaly W, Mantzoros C. Obesity as a disease. Med Clin North Am 2018;102:13-33.

[3] World Health Organization. Obesity and overweight. 2018. Available from: https://www.who.int/topics/obesity/en/ (accessed December 20, 2019).

[4] American Heart Association; American College of Cardiology; Obesity Society. Reprint: 2013 AHA/ACC/TOS guideline for the management of overweight and obesity in adults. J Am Pharm Assoc (2003) 2014;54:e3.
[5] Meldrum DR, Morris MA, Gambone JC. Obesity pandemic: causes, consequences, and solutions-but do we have the will? Fertil Steril 2017;107:833-9.

[6] Kushner RF, Kahan S. Introduction: The state of obesity in 2017. Med Clin North Am 2018;102:1-11.

[7] Kahan S, Zvenyach T. Obesity as a disease: current policies and implications for the future. Curr Obes Rep 2016;5:291-7.

[8] Després JP. Obesity and cardiovascular disease: weight loss is not the only target. Can J Cardiol 2015;31:216-22.

[9] Münzel T, Camici GG, Maack C, Bonetti NR, Fuster V, Kovacic JC. Impact of oxidative stress on the heart and vasculature. J Am Coll Cardiol 2017;70:212-29.

[10] López-Domènech S, Martínez-Herrera M, Abad-Jiménez Z, Morillas C, Escribano-López I, Díaz-Morales N, et al. Dietary weight loss intervention improves subclinical atherosclerosis and oxidative stress markers in leukocytes of obese humans. Int J Obes 2019;43:2200-9.

[11] Lévy P, Ryan S, Oldenburg O, Parati G. Sleep apnoea and the heart. Eur Respir Rev 2013;22:333-52.

[12] Senaratna CV, Perret JL, Lodge CJ, Lowe AJ, Campbell BE, Matheson MC, et al. Prevalence of obstructive sleep apnea in the general population: a systematic review. Sleep Med Rev 2017;34:70-81.

[13] Fernandes JFR, da Silva Araújo L, Kaiser SE, Sanjuliani AF, Klein MRST. The effects of moderate energy restriction on apnoea severity and CVD risk factors in obese patients with obstructive sleep apnoea. Br J Nutr 2015;114:2022-31.

[14] Dengo AL, Dennis EA, Orr JS, Marinik EL, Ehrlich E, Davy $\mathrm{BM}$, et al. Arterial destiffening with weight loss in overweight and obese middle-aged and older adults. Hypertension 2010;55:855-61.

[15] Ford ES, Greenlund KJ, Hong Y. Ideal cardiovascular health and mortality from all causes and diseases of the circulatory system among adults in the United States. Circulation 2012;125:987-95.

[16] Lloyd-Jones DM, Hong Y, Labarthe D, Mozaffarian D, Appel LJ, Van Horn L, et al. Defining and setting national goals for cardiovascular health promotion and disease reduction. Circulation 2010;121:586-613.

[17] Folsom AR, Yatsuya H, Nettleton JA, Lutsey PL, Cushman M, Rosamond WD. Community prevalence of ideal cardiovascular health, by the American Heart Association definition, and relation with cardiovascular disease incidence. J Am Coll Cardiol 2011;57:1690-6.

[18] Ford ES, Zhao G, Tsai J, Li C. Low-risk lifestyle behaviors and all-cause mortality: findings from the national health and nutrition examination survey III mortality study. Am J Public Health 2011;101:1922-9.

[19] Angelico F, Loffredo L, Pignatelli P, Augelletti T, Carnevale R, Pacella A, et al. Weight loss is associated with improved endothelial dysfunction via NOX2-generated oxidative stress downregulation in patients with the metabolic syndrome. Intern Emerg Med 2012;7:219-27.

[20] Matsudo S, Araújo T, Matsudo V, Andrade D, Andrade E, Oliveira LC, et al. Questionário Internacional de Atividade Física (IPAQ): estupo de validade e reprodutibilidade no Brasil. Rev Bras Ativ Fís Saúde 2001;6:6-18.

[21] Obesity: preventing and managing the global epidemic. Report of a WHO consultation. World Health Organ Tech Rep Ser 2000;894:1-253. 
[22] Onat A, Hergenç G, Yüksel H, Can G, Ayhan E, Kaya Z, et al. Neck circumference as a measure of central obesity: associations with metabolic syndrome and obstructive sleep apnea syndrome beyond waist circumference. Clin Nutr Metab 2009;28:46-51.

[23] Friedewald WT, Levy RI, Fredrickson DS. Estimation of the concentration of low-density lipoprotein cholesterol in plasma, without use of the preparative ultracentrifuge. Clin Chem 1972;18:499-502.

[24] Geloneze B, Repetto EM, Geloneze SR, Tambascia MA, Ermetice MN. The threshold value for insulin resistance (HOMA-IR) in an admixtured population. Diabetes Res Clin Pract 2006;72:219-20.

[25] Akselrod S, Gordon D, Ubel FA, Shannon DC, Berger AC, Cohen RJ. Power spectrum analysis of heart rate fluctuation: a quantitative probe of beat-to-beat cardiovascular control. Science 1981;213:220-2.

[26] D’Agostino RB, Vasan RS, Pencina MJ, Wolf PA, Cobain M, Massaro $\mathrm{JM}$, et al. General cardiovascular risk profile for use in primary care: the Framingham heart study. Circulation 2008;117:743-53.

[27] Grover SA, Kaouache M, Rempel P, Joseph L, Dawes M, Lau DCW, et al. Years of life lost and healthy life-years lost from diabetes and cardiovascular disease in overweight and obese people: a modelling study. Lancet Diabetes Endocrinol 2015;3:114-22.

[28] Yang Q, Zhong Y, Ritchey M, Cobain M, Gillespie C, Merritt R, et al. Vital signs: predicted heart age and racial disparities in heart age among U.S. adults at the state level. MMWR Morb Mortal Wkly Rep 2015;64:950-8.

[29] Weimin L, Rongguang W, Dongyan H, Xiaoli L, Wei J, Shiming Y. Assessment of a portable monitoring device WatchPAT 200 in the diagnosis of obstructive sleep apnea. Eur Arch Otorhinolaryngol 2013;270:3099-105.
[30] Gan YJ, Lim L, Chong YK. Validation study of WatchPat 200 for diagnosis of OSA in an Asian cohort. Eur Arch Otorhinolaryngol 2017;274:1741-5.

[31] Mahé G, Humeau-Heurtier A, Durand S, Leftheriotis G, Abraham P. Assessment of skin microvascular function and dysfunction with laser speckle contrast imaging. Circ Cardiovasc Imaging 2012;5:155-63.

[32] Sichieri R, Everhart JE. Validity of a Brazilian food frequency questionnaire against dietary recalls and estimated energy intake. Nutr Res 1998;18:1649-59.

[33] Institute of Medicine. Dietary reference intakes for energy, carbohydrate, fiber, fat, fatty acids, cholesterol, protein, and amino acids. Washington, DC: The National Academies Press; 2005, pp. 107-264.

[34] Yang Q, Cogswell ME, Flanders WD, Hong Y, Zhang Z, Loustalot F, et al. Trends in cardiovascular health metrics and associations with all-cause and CVD mortality among US adults. JAMA 2012;307:1273-83.

[35] Sabia S, Fayosse A, Dumurgier J, Schnitzler A, Empana JP, Ebmeier KP, et al. Association of ideal cardiovascular health at age 50 with incidence of dementia: 25 year follow-up of Whitehall II cohort study. BMJ 2019;366:14414.

[36] Fang N, Jiang M, Fan Y. Ideal cardiovascular health metrics and risk of cardiovascular disease or mortality: a meta-analysis. Int J Cardiol 2016;214:279-83.

[37] Andersson C, Vasan RS. Epidemiology of cardiovascular disease in young individuals. Nat Rev Cardiol 2018;15:230-40.

[38] Velasquez-Melendez G, Felisbino-Mendes MS, Matozinhos FP, Claro R, Gomes CS, Malta DC. Ideal cardiovascular health prevalence in the Brazilian population - National Health Survey (2013). Rev Bras Epidemiol 2015;18:97-108. 